\title{
SLAC-PUB-11509
}

August, 2005

\section{Ultrafast $x$-ray studies of structural dynamics at SLAC}

\author{
K.J. Gaffney ${ }^{\mathrm{a}}$, A.M. Lindenberg ${ }^{\mathrm{a}}$, J. Larsson ${ }^{\mathrm{b}}$, K. Sokolowski-Tinten ${ }^{\mathrm{c}, \mathrm{d}}$, C. Blome ${ }^{\mathrm{e}}$, O. Synnergren ${ }^{\mathrm{b}}$, \\ J. Sheppard ${ }^{\mathrm{f}}$, D.A. Reis ${ }^{\mathrm{g}}$, J.B. Hastings ${ }^{\mathrm{a}}$, the SPPS Collaboration \\ ${ }^{a}$ Stanford Synchrotron Radiation Laboratory/SLAC, Menlo Park, CA 94025, USA; \\ ${ }^{b}$ Department of Physics, Lund Institute of Technology, P.O. Box 118, S-22100, Lund, Sweden; \\ c Institut für Optik und Quantenelektronik, Friedrich-Schiller Jena, Max-Wien-Platz 1, 07743 Jena, \\ Germany; \\ d Institut für Experimentelle Physik, Universität Duisburg-Essen, D-45117 Essen, Germany; \\ ${ }^{\mathrm{e}}$ Deutsches Elektronen-Synchrotron DESY, Notkestrasse 85, 22607 Hamburg, Germany; \\ ${ }^{\mathrm{f}}$ Department of Physics, Clarendon Laboratory, Parks Road, University of Oxford, Oxford OX1, \\ 3PU, UK; \\ ${ }^{\mathrm{g}}$ FOCUS Center, Departments of Physics and Applied Physics Program, University of Michigan, \\ Ann Arbor, MI 48109, USA
}

\begin{abstract}
The melting dynamics of laser excited InSb have been studied with femtosecond x-ray diffraction. These measurements demonstrate that the initial stage of crystal disordering results from inertial motion on a laser softened potential energy surface. These inertial dynamics dominate for the first half picosecond following laser excitation, indicating that interatomic forces minimally influence atomic excursions from the equilibrium lattice positions, even for motions in excess of an $\AA$. This also indicates that the atoms disorder initially without losing memory of their lattice reference.
\end{abstract}

Keyword: femtosecond x-rays, time-resolved x-ray diffraction, non-thermal melting

Submitted to Proceedings of SPIE-The International Society for Optical Engineering 


\section{INTRODUCTION}

Ultrafast, high brightness x-ray free electron lasers (XFEL) have the potential to revolutionize the study of ultrafast transient phenomena in matter. These linear accelerator (linac) based sources will generate femtosecond (fs) $\mathrm{x}$-ray pulses with peak flux comparable to conventional lasers, and far exceeding all other x-ray sources. The Stanford Linear Accelerator Center (SLAC) has pioneered the development of linac science and technology for decades, and since 2000 SLAC and the Stanford Synchrotron Radiation Laboratory (SSRL) have focused on the development of linac based ultrafast electron and x-ray sources. This development effort has led to the creation of a new x-ray source, called the Sub-Picosecond Pulse Source (SPPS), which became operational in 2003. ${ }^{1}$ The SPPS represents the first step toward the world's first hard x-ray free electron laser (XFEL), the Linac Coherent Light Source (LCLS), due to begin operation at SLAC in 2009.

The SPPS relies on the same linac-based acceleration and electron bunch compression schemes that will be used at the LCLS to generate ultrashort, ultrahigh peak brightness electron bunches. ${ }^{2}$ This involves creating an energy chirp on the electron bunch during acceleration and subsequent compression of the bunch in a series of energy-dispersive magnetic chicanes to create $80 \mathrm{fs}$ electron pulses. The SPPS has provided an excellent opportunity to demonstrate the viability of these electron bunch compression schemes and to pursue goals relevant to the utilization and validation of XFEL light sources.

The fundamental events of chemistry, molecular biology, and materials science occur on distance and time scales governed by chemical bond lengths and vibrational periods. These chemical bond properties make x-ray science, with Ångström spatial resolution, and ultrafast laser science, with femtosecond temporal resolution, the optimal experimental tools for atomic scale investigations of structural dynamics. While standard x-ray diffraction, scattering, and spectroscopy determine the atomic structure of matter and femtosecond optical spectroscopy observes the temporal evolution of matter, their successful union has been limited by the inherently long pulse durations of synchrotrons and the low x-ray flux of ultrafast laser plasma sources. This has proven to be a critical limitation to describing important chemical, biological, and physical processes, because the inability to merge ultrafast time resolution with Ångström spatial resolution greatly limits our ability to definitively describe the structural evolution of materials. Linear electron
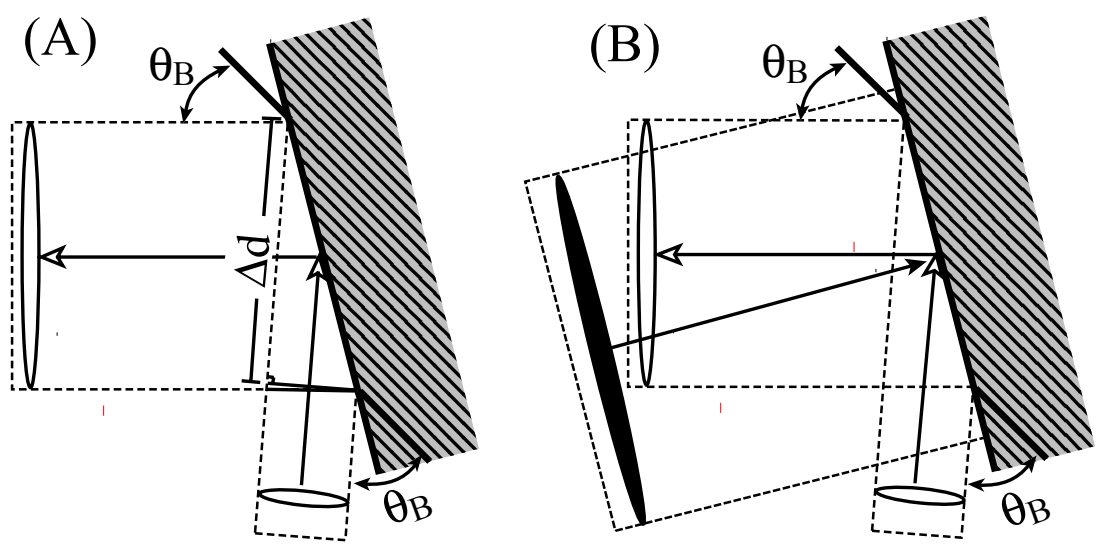

Fig. 1: (A) Asymmetric Bragg diffraction configuration. The x-ray pulse, shown as a clear oval, impinges on the InSb crystal at a grazing angle, $\theta_{g}$, and diffracts off at $2 \theta_{B}$ $\theta_{g}$ with respect to the sample surface. The clear headed arrows show the direction of propogation for the $\mathrm{x}$-ray pulse. The grazing incidence ensures that the $\mathrm{x}$-ray pulse makes a large time sweep across the crystal surface and that the x-ray penetration depth matches the laser penetration depth. (B) The laser pulse, shown as a solid black oval, approaches the surface at normal incidence. The different angles of incidence for the laser and $\mathrm{x}$-ray pulses produce a spatially dependent time delay between the laser pump and the $x$-ray probe on the crystal surface. This imprints a range of time delays on the crystal surface which can be accessed by imaging the x-ray diffraction with CCD area detector. accelerators (linac) provide an alternative approach to ultrafast hard $\mathrm{x}$-ray generation that circumvents both of these limitations. The union of these ultrafast hard $\mathrm{x}$-ray sources with ultrafast optical lasers provides a unique opportunity to observe chemical, physical, and biological phenomena with unprecedented temporal and spatial resolution.

Ultrafast studies of laser excited crystalline semiconductors provide an extensively studied phenomena that demonstrates the limitations of studying ultrafast phenomena without directly observing the atomic structure. ${ }^{3-7}$ While intense optical excitation generates metallike reflectivities consistent with the metallic liquid phase, transient reflectivities provide an indirect and inconclusive probe of atomic structure that cannot definitively signal the onset of liquid formation 
nor the structural pathway followed during the phase transformation. The interesting range of structural phenomena inferred from these optical measurements ${ }^{3-7}$ has lead to a series of ultrafast $\mathrm{x}$-ray diffraction experiments that have used an ultrafast laser to generate a plasma and produce x-ray pulses for probing structural dynamics in optical laser excited crystals. ${ }^{8-12}$ These pioneering experiments have shown crystal disordering to occur on the ultrafast time scale, including disordering faster than the time scale for energy transfer from the excited carriers to the lattice, but they have neither identified the atomic motions that lead to disordering nor determined the time needed to generate liquid-like structure and dynamics.

Experimental investigations of electronically driven melting in a semiconducting InSb crystal at the SPPS highlight the power of ultrafast $\mathrm{x}$-ray sources for studying structural dynamics with atomic detail and help validate the coming investment in XFEL facilities. Intense femtosecond (fs) laser excitation of semiconductor crystals provides a pathway to crystal melting where the time dependent evolution of the atomic structure can be monitored directly with fs x-ray diffraction. ${ }^{3-13}$ Improvements in ultrafast $x$-ray and electron sources have generated the most recent advances in our understanding of the dynamics of light driven phase transitions. While pioneering ultrafast $\mathrm{x}$-ray diffraction experiments utilizing ultrafast laser plasma sources have shown that crystals disorder faster than the rate of electron-phonon coupling, the time needed to generate liquid-like structure and dynamics remains unclear.

\section{METHODOLOGY}

These measurements relied on three critical characteristics of the experimental set-up: the 80 -fs full-width at halfmaximum (FWHM) x-ray pulse duration, ${ }^{2}$ the crossed beam x-ray-laser geometry that allows a series of relative time delays to be measured with a single pulse pair, ${ }^{14,15}$ and the low divergence of the $\mathrm{x}$-ray beam that allows for a grazing incidence x-ray geometry. ${ }^{16}$ The SPPS produces fs pulses at $8.9 \mathrm{keV}$ with $2 \times 10^{6} \mathrm{x}$-ray photons/pulse in a $200 \mathrm{x} 400 \mu \mathrm{m}$ spot in a $1.5 \%$ bandwidth. The energy spread of the $x$-ray source allows the measurement to be sensitive to changes in lattice order, but insensitive to changes in the lattice constant.

These experiments use a laser to excite the InSb crystal and the linac generated x-rays to monitor the evolution of the $\mathrm{InSb}$ structure. For these experiments to work the laser pump time of arrival at the sample must be synchronized to the arrival time of the $\mathrm{x}$-ray pulse. The electron bunches that generate the $\mathrm{x}$-rays are accelerated by a radio frequency (RF)

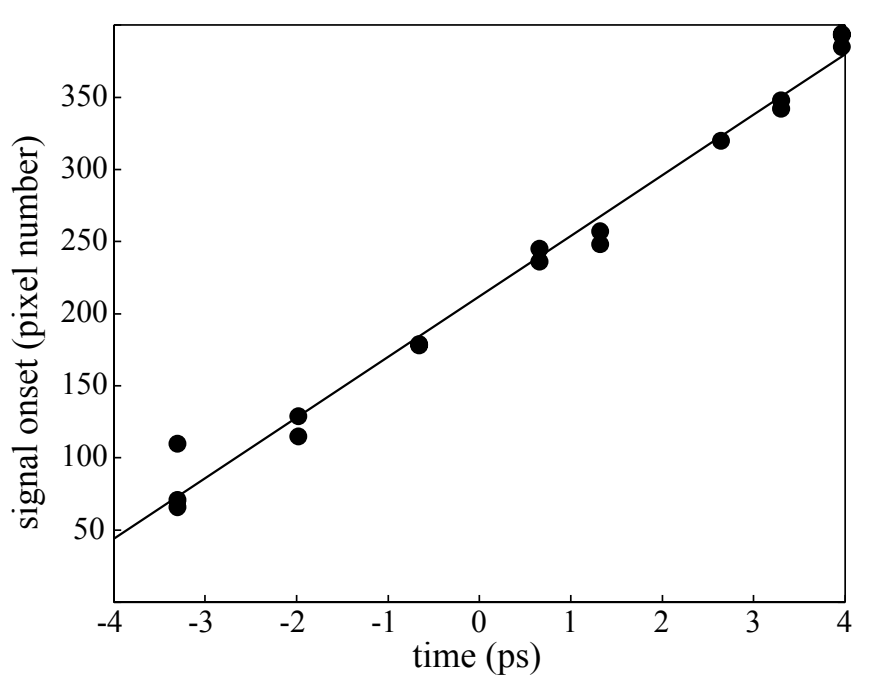

Fig. 2: Calibration of temporal axis in single-shot images for (220) reflection, obtained by recording the pixel corresponding to $\mathrm{t}=0$ for various optical delay line positions. For this geometry, each pixel corresponded to 24 fs. For the (111) reflection, the calibration corresponded to roughly $40 \mathrm{fs}$ per pixel. field. We synchronize the laser pulses to the $\mathrm{x}$-ray pulses by actively stabilizing the repetition rate of the pump laser to a $102 \mathrm{MHz}$ sub-harmonic of the linac RF. These oscillator pulses seed a Ti:Sapphire regenerative amplifier, which then pumps a multipass amplier, operating at the $10 \mathrm{~Hz}$ repetition rate of the $\mathrm{x}$-ray source. This synchronization generates a short-term timing jitter of $\sim 300 \mathrm{fs}$ rms between the $\mathrm{x}$-ray and laser pulses. $^{17}$

In order to overcome this intrinsic jitter, the relevant time evolution must be measured with a single $\mathrm{x}$-ray-laser pulse pair. We achieved this objective with a non-collinear x-ray and laser beam geometry, as shown in Fig. 1. ${ }^{14}, 15$ The different angles of incident cause the x-ray and laser pulses to sweep across the surface of the crystal with different rates. This imprints a range of pump-probe delays on the surface of the crystal, creating a spatial axis that doubles as a temporal axis. Imaging the spatial profile of the diffracted x-ray intensity with a charge-coupled 

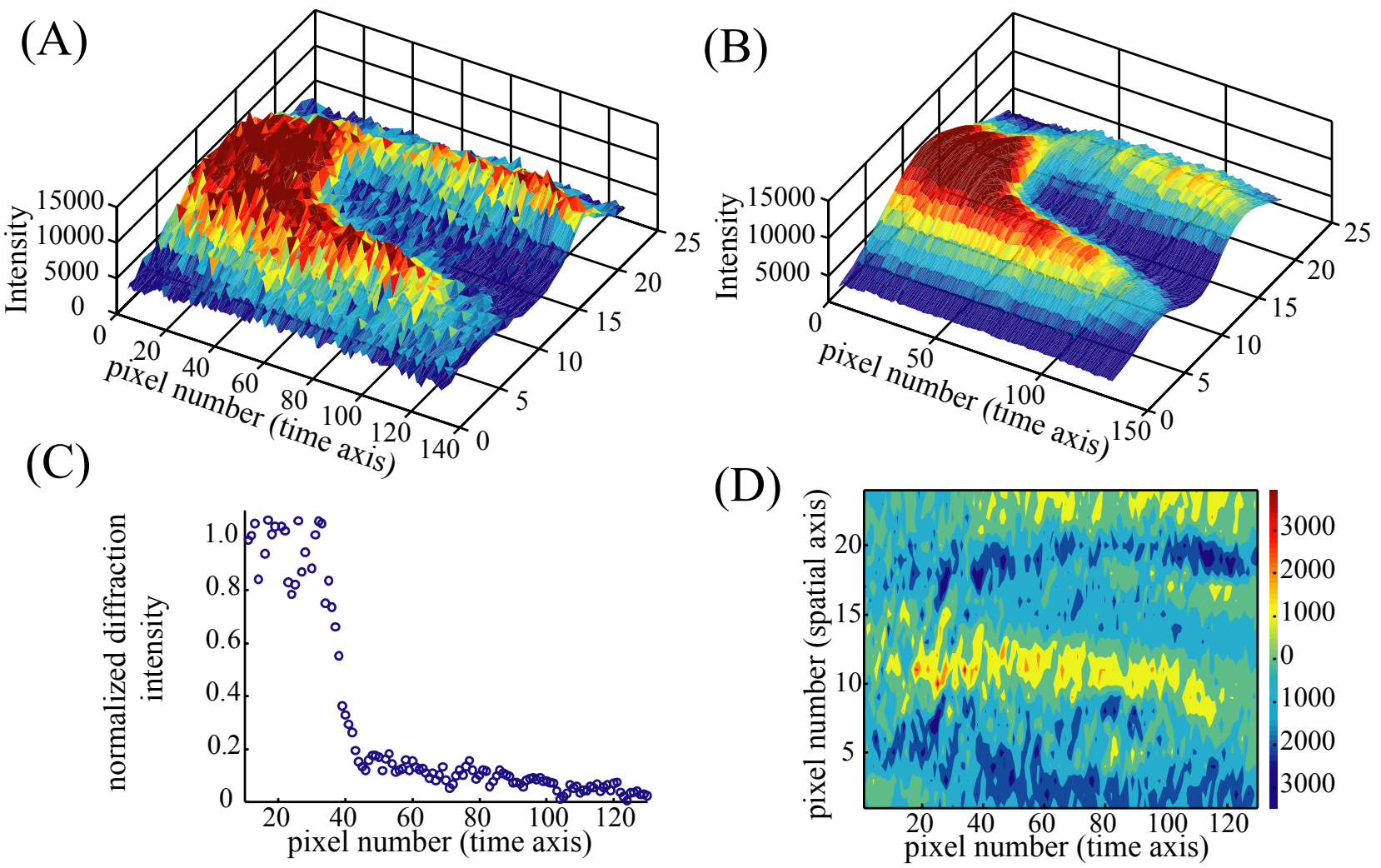

Fig. 3: (A) Two dimensional image of the x-ray diffraction for the InSb (111) Bragg peak. The sharp drop in diffracted intensity in the center of the image results from laser excitation of the crystal. The sharp edge in the signal corresponds to the spatial location on the crystal surface where the x-ray and laser had coincident arrival times. (B) the fit to the image in (A). (C) The signal extracted from the image shown in (A). Each pixel corresponds to $38 \mathrm{fs}$. (D) The difference between the fit in (B) and the signal in (A).

device $(C C D)$ area detector provides the time history around $t=0$ in a single shot. We calibrate the image by scanning the laser pump timing and measuring the spatial shift in the laser induced diffraction drop, as shown in Fig. 2.

Matching the laser and x-ray penetration represents an important requirement for successfully studying electronically driven melting. This goal has been achieved with a grazing $\mathrm{x}$-ray incidence angle of $0.4^{\circ}$ with respect to the physical surface of the asymmetrically cut InSb crystal. X-ray absorption-, not extinction-, limited penetration insures that the probe depth does not increase when the crystal disorders. At $0.4^{\circ}$ grazing incidence, $95 \%$ of the $\mathrm{x}$-ray diffraction will come from within $140 \mathrm{~nm}$ of the surface.

\section{RESULTS}

Figure 3 shows an image for the x-ray diffraction from the InSb (111) Bragg peak, as well as the two-dimensional fit of the image. We fit each row of CCD pixels transverse to the timing axis dimension to a sum of two Gaussians, one representing the x-ray diffraction intensity that would be measured without the laser and the other the laser induced drop in diffraction signal. We use the amplitudes from these two Gaussians, $A_{x-\text { ray }}(t)$ and $A_{\text {laser }}(t)$, to construct the time dependent signal, $I(Q, t)=\left(A_{x-\text { ray }}-A_{\text {laser }}\right) / A_{x-\text { ray }}$. The signal, $I(Q, t)$, extracted from a single image for the (111) Bragg peak with a Laser fluence of $130 \mathrm{mJcm}^{-2}$ appears in Fig. 3(C). Fig 3(D) shows the difference between the signal and fit. For most pixels, the error in the fit corresponds to the error expected from photon counting statistics, since the maximum number of $\mathrm{x}$-rays detected on a pixel was about 100 with each $\mathrm{x}$-ray generating 330 counts. For Poissonian 


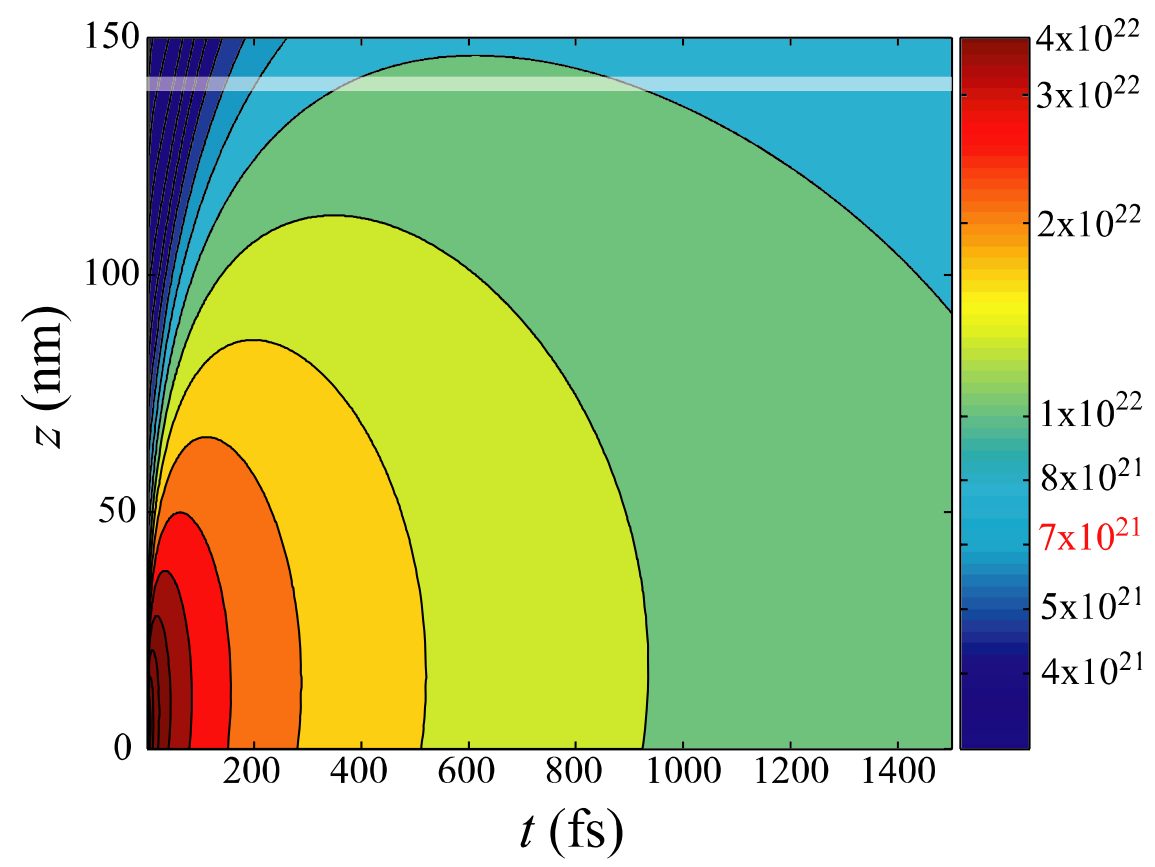

Fig. 4: Time and depth dependent electron-hole pair density. The shaded line marks a depth of $140 \mathrm{~nm}$, the depth from which $95 \%$ of the x-ray diffraction occurs. The parameters of Rousse et al. have been used to calculation the initial carrier density for a $130 \mathrm{mJcm}^{-2}$ laser fluence. Ambipolar carrier diffusion with a $150 \mathrm{~cm}^{2} \mathrm{~s}^{-1}$ diffusion constant flattens the electron-hole pair density, and by a 200 fs delay all of the x-ray probed volume has exceeded the non-thermal melting threshold of $7 \times 10^{21}$ measured by Rousse et al. These calculated densities do not account for impact ionization and Auger decay. As discussed in the text, the absorbed energy density will support electron-hole densities above the nonthermal melting threshold even after electron-hole equilibration for the time scales addressed in this letter. statistics this would correspond to a flux uncertainty of as much as $10 \mathrm{x}$ rays or roughly 3000 counts. The signal displayed in Fig. 5 results from the average of three images where the time axes have been aligned.

Before addressing the structural dynamics accessed with these measurements, the time scale of electronic equilibration, ${ }^{18,} 19$ ambipolar diffusion, ${ }^{20,}{ }^{21}$ and phonon emission must be addressed. $^{22}$ We have used the parameters of Rousse et al. to determine the laser absorption and electron-hole pair density as a function of penetration depth. ${ }^{9}$ Electronic excitation will be followed by carrier diffusion and equilibration. For the large electronhole densities generated in these studies, carrier diffusion occurs with ambipolar diffusion constants $>100$ $\mathrm{cm}^{2} \mathrm{~s}^{-1} .^{20,21}$ This diffusion smoothes the distribution of electron-hole pairs on a time scale faster than the rate of inertial structural dynamics. This insures that the excitation density created with the $130 \mathrm{mJcm}^{-2}$ fluence exceeds the non-thermal melting threshold of $7 \times 10^{21}$

determined by Rousse et al. within the sample volume probed by the x-ray pulse. ${ }^{9}$ The time and depth dependent carrier density for a $120 \mathrm{mJcm}^{-2}$ pulse and an ambipolar diffusion constant of $150 \mathrm{~cm}^{2} \mathrm{~s}^{-1}$ appears in Fig. 4.

Auger decay and impact ionization will also change the excitation density in the excited volume. While the rates of these processes have not been measured at the electron-hole pair densities generated in this study, theory and experiment indicate that the Auger relaxation takes tens to hundreds of fs at very high carrier densities. ${ }^{18,19}$ The carrier density after electronic equilibration should still exceed the theoretical and measured non-thermal melting threshold of roughly $7 \times 10^{21} \mathrm{~cm}^{-39,13,23}$ because the energy absorbed by the electrons in the first $150 \mathrm{~nm}$ of InSb when irradiated with a $130 \mathrm{mJcm}^{-2}$ laser pulse supports an average equilibrium electron-hole pair concentration of $9 \times 10^{21} \mathrm{~cm}^{-3}$. This has been calculated using a simple approximation to the InSb conduction band density of states. ${ }^{24}$ Cooling of the electronic system will be comparatively slow because the optical phonon emission time for electrons in the $\Gamma$-conduction band occurs with an average rate of $\sim 3$ ps for the energy range that will be populated by the laser pump pulse. ${ }^{22}$ Consequently, the magnitudes and rates for the different processes observed in the diffraction experiment for time delays up to $3 \mathrm{ps}$ correspond to the dynamics of a fairly uniformly excited sample with an electronic temperature upwards of $10000 \mathrm{~K}$ and a lattice temperature less than the $798 \mathrm{~K}$ melting point of InSb.

Figure 5(A) shows the time dependent x-ray diffraction intensity from a laser excited crystal for both the (111) and (220) reflections. The diffracted intensity decays non-exponentially and can be well fit to a Gaussian, $I(Q, t)=\exp \left(-t^{2} / \tau^{2}\right)$ for $0<t<500 \mathrm{fs} .{ }^{16}$ As shown in Figure 5(A), the (220) Bragg peak decays faster than the (111).

The fit gives time constants, $\tau$, of 280 and 430 fs for the (220) and (111) reflections. The ratio of these time constants, $\tau_{111} / \tau_{220}=1.5 \pm 0.2$, equals the ratio of the magnitude of the reciprocal lattice vectors for the two reflections $(\sqrt{8 / 3})$. 

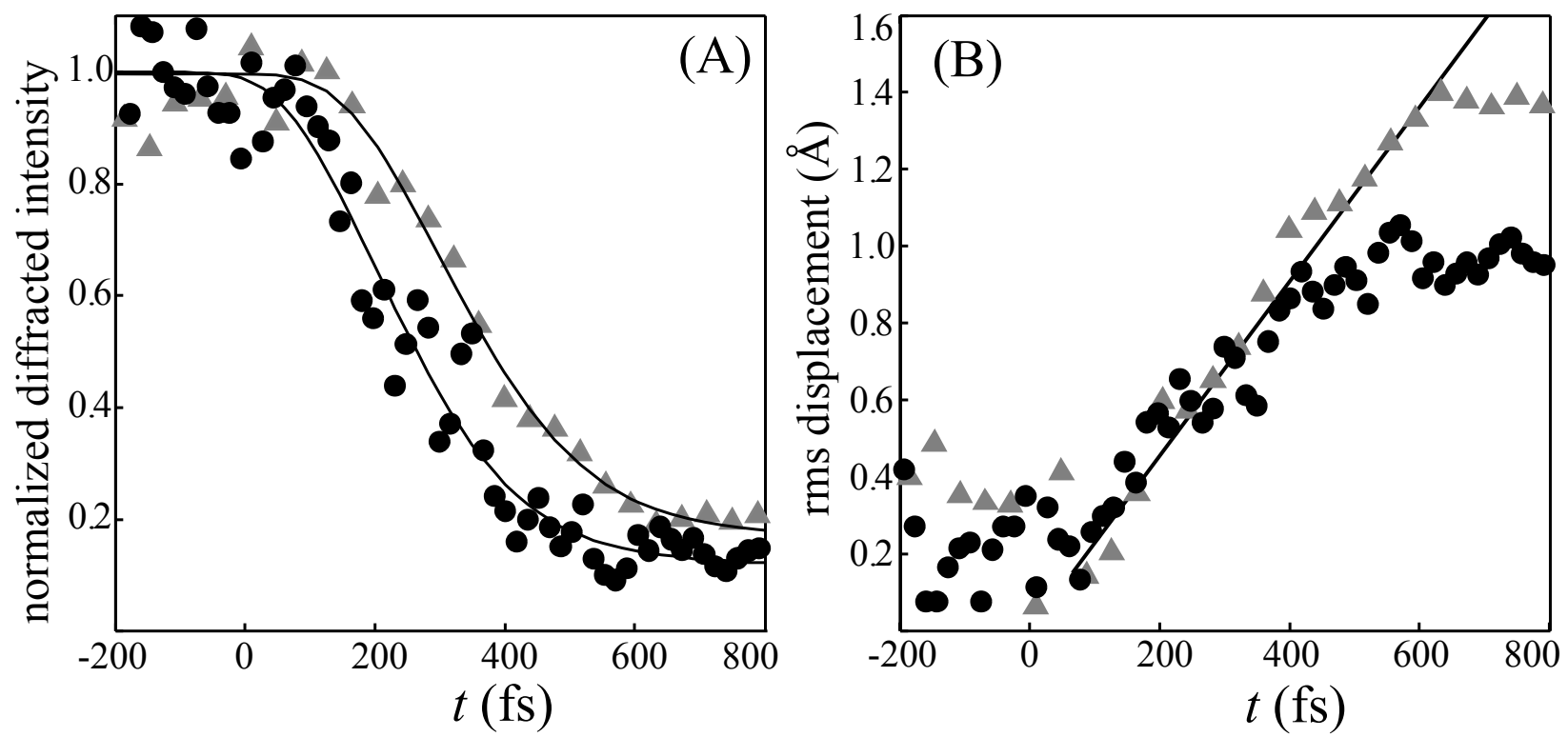

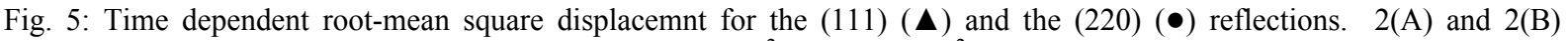
correspond to data collected with laser fluences of $130 \mathrm{mJcm}^{-2}$ and $50 \mathrm{mJcm}^{-2}$, respectively.

This inverse- $Q$-dependent scaling and Gaussian time dependence strongly implies statistical, atomic motion that can be described using a time-dependent Debye-Waller-like model that relates the time dependent decrease in diffracted intensity to a time-dependent root mean square (rms) displacement, $I(Q, t)=\exp \left(-Q^{2}\left\langle u^{2}(t)\right\rangle / 3\right)$, where $Q$ is the reciprocal lattice vector and $\left\langle u^{2}(t)\right\rangle$ is the time-dependent mean-square displacement of the photo-excited atoms, averaged spatially over the sample. ${ }^{16}$ The time dependent root mean square (rms) displacement appears in Figure 5(B) for the (111) and the (220) reflections. For the first few hundred fs, the rms displacement in these two directions have identical time dependence and increase linearly with time. This linear time dependence demonstrates that the initial disordering results from inertial atomic motion on the laser modified potential. The momentum of the ions does not change upon laser excitation, in accordance with the Franck-Condon principle. Thus the atoms will initially sample the new potential energy surface with a velocity distribution dictated by the lattice temperature prior to laser excitation. This predicts the rms displacement should increase linearly with time, with an average velocity determined by the average atomic mass and the temperature. For InSb at room temperature, the predicted slope of $\left(3 k_{B} T / M\right)^{1 / 2}=2.5 \AA / p s$ agrees reasonably well with the experimental value of $2.3 \pm 0.2 \AA / \mathrm{ps}$.

The amplitude of the inertial response indicates the magnitude of the lattice softening, since the lattice temperature remains constant during inertial motion and the rms displacement is inversely proportional to the average frequency of the thermally excited phonons. The inertially accessible rms displacement exceeds the ground state rms displacement of $0.16 \AA$ by nearly an order of magnitude, as shown in Figure 5(B). Interestingly, these large displacements that occur during the first few hundred fs result in an increased disorder, but not a transition to a liquid like structure because these inertial motions preserve the atomic memory of the initial lattice configuration. Only after velocity randomizing collisions can the structure begin to lose translational symmetry.

X-ray free electron lasers possess the capacity to transform the study of dynamic phenomena in biology, chemistry, and physics. The Sub-Picosecond Pulse Source has provided an opportunity to demonstrate how an ultrafast hard x-ray source with high peak brightness can clarify the evolution of atomic structure during a physical or chemical transformation with unprecedented detail ${ }^{16}$. 


\section{ACKNOWLEDGEMENTS}

The SPPS has been the product of an international collaboration supported by the SLAC/SSRL, ANL/APS, BNL/NSLS, LLNL, HASYLAB/ DESY, and ESRF laboratories and synchrotron facilities and the University of Chicago/BIOCARS, the University of California at Berkeley, the University of Michigan, Copenhagen University, Uppsala University, Chalmers University, and Lund University. The SPPS collaboration in total has relied on the contributions of more than 50 scientists. Portions of this research were supported by the U.S. Department of Energy, Office of Basic Energy Science through direct support for the SPPS, as well as individual investigators and SSRL, a national user facility operated by Stanford University. Additional support for the construction of SPPS was provided in part by Uppsala University and the Swedish Research Council. Scientists within the collaboration have relied on financial support by the Deutsche Forschungsgemeinschaft, the European Commission through the FEMTO, X-RAY FEL PUMP-PROBE and XPOSE projects, the Wallenberg Research Link, and The Swedish Foundation for Strategic Research.

\section{REFERENCES}

1. M. Cornacchia, et al., A Sub-Picosecond Photon Pulse Facility for SLAC (SLAC-PUB-9850, 2001)

2. P. Krejcik, et al., in Proceeding of the 2003 Particle Accelerator Conference, edited by J. Chew, S. Lucas and S. Webber (IEEE, Piscataway, NJ, IEEE, Portland, OR, 2003), p. 423.

3. C. V. Shank, R. Yen and C. Hirlimann Time-resolved reflectivity measurements of femtosecond-optical-pulse induced phase-transitions in Silicon. Phys. Rev. Lett. 50, 454-457 (1983).

4. P. Saeta, et al. Ultrafast electronic disordering during femtosecond laser melting of GaAs. Phys. Rev. Lett. 67, 1023-1026 (1991).

5. K. Sokolowski-Tinten, J. Bialkowski and D. von der Linde Ultrafast laser-induced order-disorder transitions in semiconductors. Phys. Rev. B 51, 14186-14198 (1995).

6. A. M. T. Kim, J. P. Callan, C. A. D. Roeser and E. Mazur Ultrafast dynamics and phase changes in crystalline and amorphous GaAs. Phys. Rev. B 66, (2002).

7. S. K. Sundaram and E. Mazur Inducing and probing non-thermal transitions in semiconductors using femtosecond laser pulses. Nature Mater. 1, 217-224 (2002).

8. C. W. Siders, et al. Detection of nonthermal melting by ultrafast X-ray diffraction. Science 286, 1340-1342 (1999).

9. A. Rousse, et al. Non-thermal melting in semiconductors measured at femtosecond resolution. Nature 410, 65-68 (2001).

10. K. Sokolowski-Tinten, et al. Femtosecond x-ray measurement of ultrafast melting and large acoustic transients. Phys. Rev. Lett. 87, 225701 (2001).

11. C. Rose-Petruck, et al. Picosecond-milliangstrom lattice dynamics measured by ultrafast x-ray diffracton. Nature 398, 310312 (1999)

12. A. M. Lindenberg, et al. Time-resolved x-ray diffraction from a coherent phonons during a laser-induced phase transition. Phys. Rev. Lett. 84, 111-114 (2000).

13. P. Stampfli and K. H. Bennemann Time-dependence of the laser-induced femtosecond lattice instability of Si and GaAs role of longitudinal optical distortions. Phys. Rev. B 49, 7299-7305 (1994).

14. R. Neutze and J. Hajdu Femtosecond time resolution in x-ray diffraction experiments. Proc. Natl. Acad. Sci. USA 94, 56515655 (1997).

15. O. Synnergren, et al. Projecting picosecond lattice dynamics through x-ray topography. App. Phys. Lett. 80, 3727-3729 (2002).

16. A. M. Lindenberg, et al. Atomic-Scale Visualization of Inertial Dynamics. Science 308, 392-395 (2005).

17. A. L. Cavalieri, et al. Clocking femtosecond x rays. Phys. Rev. Lett. 94, 114801 (2005).

18. E. J. Yoffa Dynamics of Dense Laser-Induced Plasmas. Phys. Rev. B 21, 2415-2425 (1980).

19. P. M. Fauchet The Auger Rate in Highly Excited Indium-Antimonide. Phys. Status Solidi B 110, K11-K15 (1982).

20. J. F. Young and H. M. van Driel Ambipolar diffusion of high-density electrons and holes in Ge, Si, and GaAs - many-bdy efects. Phys. Rev. B 26, 2147-2158 (1982).

21. H. Bergner, V. Bruckner and M. Schubert On the Investigation of the Diffusion-Processes of Photoexcited Carriers in Silicon by Ps-Reflectivity Measurements. J. Lumin. 30, 114-119 (1985).

22. T. Grave, E. Scholl and H. Wurz Optically Induced Avalanche in Insb. J. Phys. C 16, 1693-1711 (1983).

23. R. Biswas and V. Ambegaokar Phonon spectrum of a model of electronically excited silicon. Phys. Rev. B 26, 1980-1988 (1982). 
24. J. R. Chelikowsky and M. L. Cohen Nonlocal Pseudopotential Calculations for Electronic-Structure of 11 Diamond and Zincblende Semiconductors. Phys. Rev. B 14, 556-582 (1976). 